\title{
Work Engagement in Nursing: A concept Analysis
}

\author{
Safyia S. Sayed ${ }^{1}$, Manal Z. Ahmed ${ }^{2}$, Manal M. Bakr ${ }^{3}$, and Nabila M. \\ Sherief $^{4}$ \\ ${ }^{1}$ Assistant lecturer of Nursing Administration, ${ }^{2}$ Professor of Nursing Administration, \\ ${ }^{3}$ Assistant Professor of Nursing Administration, ${ }^{4}$ Lecturer of Nursing Administration \\ Faculty of Nursing, Menoufia University
}

\begin{abstract}
Work engagement is ongoing challenge among nurse leaders, including health care systems, to keep nurses engaged. Conceptual clarity about work engagement gives empirical direction for future research and a theoretical underpinning for the myriad studies about nurses and their work environment. Purpose: The present study was conducted with the aim of clarifying and defining the concept of work engagement, its attributes, antecedents, and consequences. Methods: In this study, the steps of concept analysis were as follows: Select a concept, determine the aims of the analysis, identify various definitions of the concept, determine the concept attributes and identify the antecedents and consequences. All studies between the years 2010 and 2019 were reviewed. For purposes of this concept analysis, PubMed, Google search engines, Ovid, and ProQuest, were scanned and searched using the keywords. Conclusion: Work engagement is a positive, fulfilling state of mind about work that is characterized by vigor, dedication and absorption. Trust (managerially, organizationally and collegially) and autonomy are the antecedents of work engagement. The outcomes of nurses' work engagement are higher levels of personal initiative that are contagious, decreased hospital mortality rates and significantly higher financial profitability of organizations. There is a need to explore the determinants that encourage work engagement among nurses. Also there is a need to explore the influence of external work environment on nursing work engagement.
\end{abstract}

Keywords: Autonomy, concept analysis, nurses work engagement, trust.

\section{Introduction}

Work engagement in nursing is becoming strategically important as three important factors converge: a global shortage of nurses who are the largest group of healthcare providers; political resolve to restrain the growth of rising healthcare costs in industrialized nations; and a medical error rate that threatens the health of nations. While the concept of work engagement emerges from the new 'positive psychology' (that focuses on human strengths, rather than limitations, work engagement has captured global research attention because it is amenable to change (Fasoli, 2010).

Clarifying the concept of work engagement is important in nursing because as noted, The danger with concepts like engagement is that they can become unwieldy, fuzzily-defined terms invoked as panaceas for the dilemmas of workforce management nursing review of the research on work engagement concluded that there is an essential need to differentiate the antecedents from defining attributes because these have been interchangeably used. This conceptual confusion has prompted four distinctive lines of research: personal engagement, burnout/engagement, work engagement and employee engagement (Bargagliotti, 2012).

\section{Purpose}

The present study was conducted with the aim of clarifying and defining the concept of work engagement, its attributes, antecedents, and consequences.

\section{Methods}


The aim of concept analysis is to examine the basic elements of a concept. The process allows researchers to distinguish between similarities and differences between concepts. The concept analysis method helps to clarify concepts used in nursing practice that have a bro ad scope (Walker \& Avant, 2011). In this study, the steps of concept analysis were as follows: Select a concept, determine the aims of the analysis, identify various definitions of the concept, determine the concept attributes and identify the antecedents and consequences.

\section{Data Collection}

For purposes of this concept analysis, PubMed, Google search engines, Ovid, and ProQuest, were scanned. These databases were searched for the keywords "work engagement ", " engagement", and "work engagement in nursing.". All studies between the years 2010 and 2019 were reviewed. Inclusion criteria were: written in English, and described or studied work engagement in any setting with any population.

\section{Concept of Work Engagement}

Work engagement is defined as a 'positive, fulfilling work-related state of mind' and well-being at work that is 'characterized by vigor, dedication, and absorption (Schaufeli et al., 2010).

Work engagement vary, it is widely agreed that work engagement is exhibited as a high level of energy in one's work, or vigor, and identification with one's work (Bakker et al., 2011). It is important to note that work engagement extends beyond individual or immediate situations, which may be described by other forms of engagement, such as patient engagement, or therapeutic engagement, to an overall psychological state towards one's work (Leiter and Bakker, 2010).

Vinje and Mittlemark's (2008) qualitative study of community health nurses' work engagement defined work engagement as 'searching for, experiencing, and holding on to the meaningful work that enables one to live one's values'.

The concept of nurse engagement is often used to describe nurses' commitment to and satisfaction with their jobs. In reality, these are just two facets of engagement. Additional considerations include nurses' level of commitment to the organization that employs them, and their commitment to the nursing profession itself (Dempsey \& Reilly, 2016).

Nurse engagement is defined as a persistent and pervasive affective cognitive state of mind characterized by psychological presence, energy and involvement (Gustavsson, 2010) with a heightened dedication to the work environment and organization (Jenaro, Flores, Begonia \& Cruz, 2010).Staff nurse work engagement can be preceded by organizational factors such as authentic leadership style and a supportive work environment; which may include access to job resources, control over workloads, fairness, rewards, and developmental opportunities (Bogaert, Clarke, Willems \& Mondelaers, 2012\& Bamford et al., 2012).The impact of work engagement has been investigated with suggestions for increased job satisfaction, quality of work life, and organizational commitment (Jenaro et al., 2010).As leaders in hospital environments, nurse managers are optimally positioned to enhance nurse's engagement (Bamford et al., 2012).

\section{Attributes of Work Engagement}

The defining attributes are those characteristics of the concept that both define and differentiate the concept (Walker \& Avant, 2011). Work engagement is a fulfilling, positive work related state of mind characterized by three main components: dedication, absorption, and vigor. Each of these components is defined based on the impact they have on the individual in their work. Vigor is characterized by the mental 
resilience and high levels of energy experienced during work. It is the willingness to exert effort in ones work and to persist during difficult times. Dedication is characterized by strong involvement in work. It is the experience of enthusiasm, inspiration, pride, sense of significance, and challenge. Absorption is characterized by the happy engrossment in ones work, the full level of concentration where time quickly passes and one has difficulty detaching from work (Schaufeli \& Salanova, 2011).

Wong et al., 2010, described three interrelated attributes of nurses' work engagement: having a 'calling' which provides the path to meaningfulness; zest which happens when experiencing meaningfulness in work; and vitality, which is the ability to hold onto meaningfulness in work. The two most commonly agreed on dimensions of work engagement are high levels of energy and identification with work. Vigor, absorption and dedication were selected as defining attributes of work engagement.

Job Demands-Resources (JD-R) Model of Work Engagement

The Job Demands-Resources (JD-R) model was first introduced by Demerouti and her colleagues in 2001, and to date is the most commonly used theoretical framework in studies on work engagement (Hakanen \& Roodt, 2010). A key assumption of the model is that each job or occupation has its own risk factors related to job stress, and therefore the model can be adapted and applied to various occupational settings. The JD-R model outlines two specific sets of working conditions. Job demands and job resources are related to negative and positive outcomes.

The original JD-R model includes a dual process, through which job demands initiate a health impairment process leading to negative health-related outcomes, and job resources initiate a motivational process through which positive performance-related outcomes are realized (Demerouti et al., 2001\&Schaufeli \& Bakker, 2004). Job demands refer to "physical, social, or organizational aspects of the job that require sustained physical and/or psychological effort or skills and are associated with certain physiological and/or psychological costs". Alternately, job resources refer to the "physical, psychological, social, and organizational aspects of the job that are either functional in achieving work goals, reduce job demands and the associated physiological and psychological costs, or stimulate personal growth, learning, and development' (Demerouti et al., 2001).

Hakanen \& Roodt (2010) concluded that job resources are the most important factors in predicting work engagement and accordingly, the most important factors for healthcare organizations to improve employee performance. On other hand, personal resources, such as self-efficacy, self-esteem, and optimism were added to the model because research demonstrated that they are both related to job resources and independent predictors of work engagement (Hakanen \& Roodt, 2010).In a recent summary of research on the concept across disciplines, Demerouti \& Bakker (2011) concluded that JD-R model of engagement should be used when considering interventions to promote engagement because it offers clear and valuable implications for practice. 


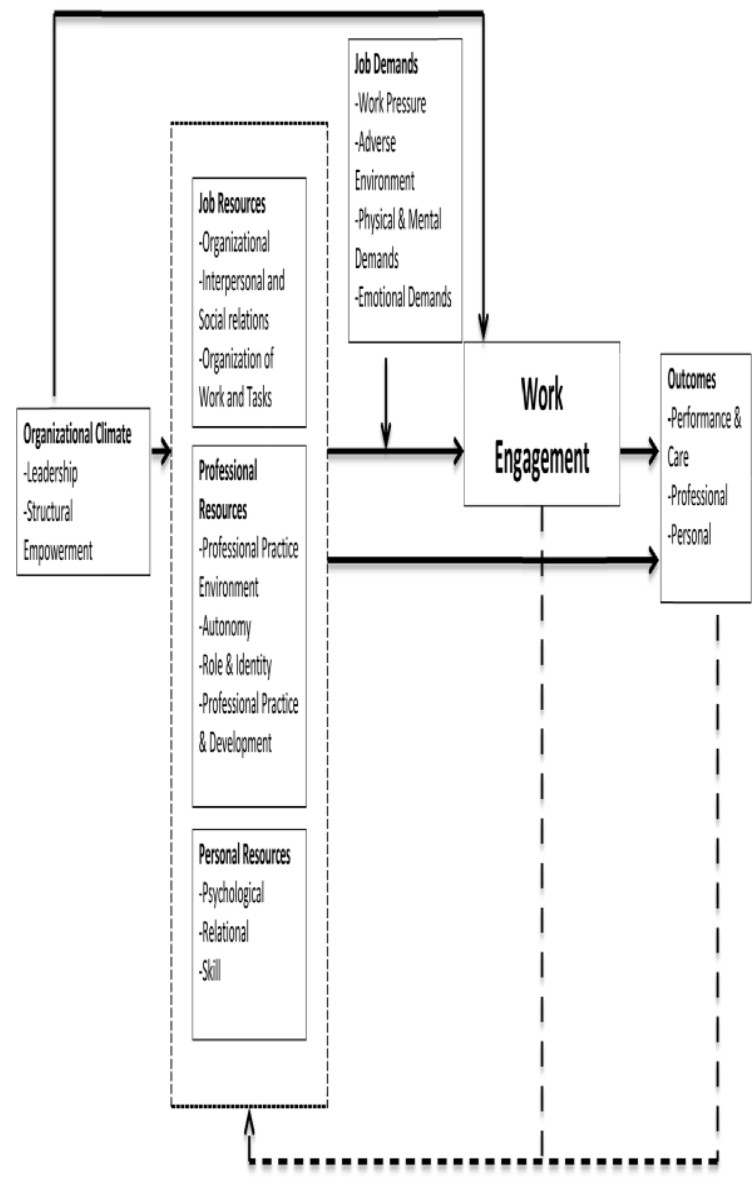

Nursing job demand-resources (NJD-R) model of engagement in professional nursing practice (Demerouti et al., 2001).

\section{Benefits of Work Engagement}

Benefits of work engagement for healthcare organizations are: reducing nurses' absenteeism, improving nurses' morale, increasing safety, more capability for the use of local volunteers by using succession plans, reducing nurses' turnover, and increasing the nurses' motivation. Moreover, benefits outside healthcare organizations are: increasing productivity, increasing profit and revenue, increasing customer loyalty, and increasing the ability to attract talented staff from outside the organization. In addition, increasing staff productivity, higher wages, increasing self-esteem, and improving the level of nurse's health are the positive effects of work engagement on employees (Dalenjan, Shoorideh, Hosseini, \& Mohtashami, 2017).

\section{Differences in Drivers of Nurse Engagement}

An analysis of Press Ganey's national nurse engagement database (Dempsey,Reilly and Noble 2015) also identified drivers of nurse engagement. Items were selected in decreasing order of beta (standardized regression coefficient) from the pool of items with $66 \%$ response rate or higher. The key drivers of nurse engagement in 2015 included, this organization provides high-quality care and service, this organization treats employees with respect, like the work I do, the environment at this organization makes employees in my work unit want to go above and beyond what's expected of them, my pay is fair compared to other healthcare employers in this area, my job makes good use of my skills and abilities, I get the tools and resources, i need to provide the best care/service for our clients/patients, this organization provides career development opportunities, this organization conducts business in an ethical manner, and patient safety is a priority in this organization.

\section{Factors Influencing on Work Engagement}

Influencing factors on work engagement are categorized into six themes: organizational climate, job resources, professional resources, personal resources, job demands, and demographic variables (Keyko, Cummings, Yonge \& Wong, 2016).First, Organizational climate: influencing factors that can be considered part of the broad organizational climate encompassing two categories, leadership and structural empowerment. Leadership is a specific approach to leadership, authentic leadership and transformational leadership (Hayati et al., 2014), are both reported to influence work engagement directly or indirectly (Bamford et al., 2013). 
Second, Job resources: Job resources are the most frequently studied influencing factors on work engagement. Job resources factors are grouped into three sub-themes, interpersonal and social relations, organizational and organization of work and tasks. Predictors of work engagement included social identification with the work unit, satisfaction with interaction, relational coordination, and collaboration with physicians (Sawatzky \& Enns, 2012). Also, relationships with nurse peers, patients, and families, and support from peers, family, and management influenced work engagement (Wu, 2010). Organization of work and tasks: Rewards, fairness, and staffing resources (Sawatzky \& Enns, 2012).

Third, professional resources: Professional resources enable nurses to engage in nursing practice according to standards and scope of practice allowing them to meet professional goals. Professional resources are the second most common influencing factors of nurses work engagement. Professional resources factors are categorized into four subthemes: professional practice environment, autonomy, role and identity, and professional practice and development (Keyko, Cummings, Yonge and Wong, 2016).

Fourth, Personal resources factors are grouped into three sub-themes including: psychological, relational, and skill. Psychological factors include psychological capital, psychological empowerment, self-transcendence, and turnover cognitions, which are positive predictors of work engagement. However, turnover cognitions, including thinking of quitting, job-search behavior, intent to quit, and intent to search, are negative predictors of work engagement (Wang and Liu, 2013; Laschinger et al., 2014\&Simpson, 2010).

Fifth, Job demands factors are categorized as work pressure, physical and mental demands, emotional demands, and adverse environment. Work pressure. There are three factors related to work pressure such as: workload, indirect patient care, and adjustment to nursing work) have negative impact on nurses' work engagement (Byrne, Peters \& Weston, 2016).

Factors relevant to physical and mental demands, including shiftwork, day shift versus night shift, length of shift, hours worked per week, recovery and rest, and no occupational care provision. No occupational care provision also affects work engagement (Sawatzky \& Enns, 2012). Shiftwork considered negative predictor of work engagement whereas results for day shift were equivocal (Rivera et al., 2011). Hours of work per week is related to work engagement while length of work shift, recovery and rest are also related to engagement (Simpson, $2009 \& \mathrm{Wu}, 2010)$.

\section{Antecedents}

Antecedents are those factors that precede the occurrence of the concept (Walker \& Avant, 2011). When the findings from work engagement studies are sifted through the evidence about the practice environment of nurses (Institute of Medicine 2003, Joint Commission for Accreditation of Healthcare Organizations trust and autonomy emerge as the two antecedents to the work engagement of professional nurses (Cummings et al., 2010).

\section{Autonomy}

Autonomy is a threshold issue for professional nursing practice (Chen \& Johantgen,2010). Autonomy is an abstract and complex concept, with which individuals gain the capacity to make an informed and independent decision in order to achieve the desired outcome. Autonomy is a core ethical principle in the field of healthcare. Nursing professional autonomy is a complex, multi-dimensional concept and the basis of 
professionalism (Asakura, Satoh \& Watanabe, 2016). Autonomy described as self-direction that leads to work engagement (Bargagliotti, 2012).

\section{Trust}

Trust, the second antecedent of work engagement in nursing, is salient for nurses because integrity is legally demanded and central to the ethical comportment of nurses. Subsequently, trust is a fundamental expectation that nurses have of their practice setting (Bargagliotti, 2012).

Empathy and trust are critical to creating a positive work environment. Trust is built on accountability, integrity, and fidelity at all levels of the organization. Consistency and empowerment that have been so foundational for shared governance help to instill trust in leadership and colleagues. Empathy, not only for patients but also for caregivers means treating one another with respect and anticipating others' needs. This empathy and trust for each other and the organization must be embedded within the culture of the organization for it to be tangible in the care (Vegsund, 2014). Work engagement requires creating a culture of trust at all organizational levels

\section{Consequences of Work Engagement}

There are organizational and personal consequences of the work engagement of nurses outcomes of work engagement are categorized into: personal outcomes, performance and care outcomes, and professional outcomes (Keyko, Cummings, Yonge \& Wong, 2016).Personal outcomes refer to the effects of work engagement on the individual nurse. Personal outcomes are the most commonly examined outcomes of work engagement. Greater work engagement increase job satisfaction, compassion satisfaction and career satisfaction and decrease burnout, job turnover intent, and intention to leave current position (Sawatzky \& Enns, 2012\& Laschinger, 2012).

Performance and care outcomes refer to various aspects of nurses' performance, organizational outcomes and patient outcomes. These include voice behavior, perceived care quality, quality care, work effectiveness, patient satisfaction, adverse events, and productivity (Wong et al., 2010).

Professional outcomes impact the broader professional body of nurses. They can be differentiated from organizational outcomes because they are not limited to a single organization. Presence of work engagement among work environments resulting in more employee productivity, increasing job satisfaction, quality of work life, and organizational commitment (Jenaro, Flores, Orgaz \& Cruz, 2010).

\section{Conclusion}

Creating practice environments that fully engage nurses in their practice is a central issue for the nursing profession. Safety issue for patients is important economic issue for all the nations. Since the antecedents of work engagement are relational rather than transactional, they have no financial costs. These antecedents shed important light on the direction that healthcare organizations, nurse managers and nurses can take to create a work environment that supports the work engagement of nurses. This concept analysis provides a clearer direction for future research in nurses' work engagement.

\section{Implications for Practice}

- The work engagement of nurses is enhanced in trustworthy practice settings that value the autonomy of nurses.

- The antecedents for work engagement are relational ways of behaving and being, rather than resources that can be transacted. 
- Safe patients' care require the engagement of nurses in their practices.

\section{References}

Asakura, K., Satoh, M., \& Watanabe, I. (2016).The development of the attitude toward professional autonomy scale for nurses in Japan: Psychol Rep, 119 (3) ,761-782

Bamford, M., Wong, C. A., \& Laschinger, H. (2013). The influence of authentic leadership and areas of work life on work engagement of registered nurses. Journal of Nursing Management, 21(3),529-540.

Bargagliotti, L.A. (2012). Work engagement in nursing: a concept analysis. Journal of Advanced Nursing, 68(6), 1414-1428. doi: 10.1111/j.1365-2648.2011.05859.x.

Byrne, Z. S., Peters, J. M., \& Weston, J. W. (2016). The struggle with employee engagement: Measures and construct clarification using five samples. Journal of Applied Psychology. Advance online publication.

doi: 10.1037/ap10000124.

Chen, Y.-M., \& Johantgen, M.E. (2010) Magnet hospital attributes: a in European hospitals: A multilevel model of job satisfaction. International Journal of Nursing Studies, 47, 1001-1012. doi:10.1016/j.ijnurstu.2009.12.016.

Demerouti, E., \& Bakker, A. B. (2011). The job demands-resources model: Challenges for future research. SA Journal of Industrial Psychology, 37(2), 1-9.

Demerouti, E., Bakker, A. B., De Jonge, J., Janssen, P. P., \& Schaufeli, W. B. (2001). Burnout and engagement at work as a function of demands and control. Scandinavian Journal of Work, Environment \& Health, 279286.
Dempsy, C., \&Reilly, B. (2016).Nurse engagement what are the contributing factors for success. On line journal of issue in nursing. (21) $1-4$.

[9] Dempsey, C., Reilly, B.A., \& Noble, B. (2015). Strengthening culture: Nurse engagement insights to inform HR strategies [Webinar]. Retrieved from http://www.pressganey.com.

Fasoli, D.J. (2010). The culture of nursing engagement: a historical perspective. Nursing Administration Quarterly 34(1),18-29.

Hakanen, J.J., Roodt, G., (2010). Using the job demands-resources model to predict engagement: analyzing a conceptual model. In: Bakker, A.B., Leiter, M.P (Eds.), Work Engagement: A Handbook of essential theory and research Psychology Press, New York, NY, pp. 85-101 .

Hayati, D., Charkhabi, M., Naami, A., (2014). The relationship between transformational leadership and work engagement in governmental hospitals nurses: a survey study. SpringerPlus, 3 (25), http:// dx.doi.org/10.1186/2193-1801-3-25.

Hosseinpour-Dalenjan, L., AtashzadehShoorideh, F., Hosseini, M., \& Mohtashami, J. (2017). The Correlation Between Nurses' Work Engagement and Workplace Incivility. Iranian Red Crescent Medical Journal, 19(4).

Jenaro, C., Flores, N., Orgaz, M.B., \& Cruz, M. (2010) Vigor and dedication in nursing professionals: towards a better understanding of work engagement. Journal of Advanced Nursing, 67(4), 865-875.

Keyko, K.(2014). Work engagement in nursing practice: a relational ethics perspective. Nurs Ethics, 
http://dx.doi.org/10.1177/

0969733014523167.

Laschinger, H. K., Wong, C., Gummings, G., \& Grau, A. (2014). Resonant Leadership and Workplace Empowerment: The Value of Positive Organizational Cultures in Reducing Workplace Incivility. Nursing Economics , 32 (1), 5-14.

Laschinger, HK. (2012). Job and career satisfaction and turnover intentions of newly graduated nurses. J Nurs Manag,20(4),472-484.

Leiter, M.P., Bakker, A.B.(2010). Work engagement: introduction. In: Bakker, A.B., Leiter, M.P. (Eds.), Work Engagement: A Handbook of Essential Theory and Research. Psychology Press, New York, NY,. 10-24.

Li, I., Kuo, H., Huang, H., Lo, H., \& Wang, H. (2013). The mediating effects of structural empowerment on job satisfaction for nurses in longterm care facilities. Journal of Nursing Management. 21(3); 440-8.

Multi-level model of job satisfaction. International Journal of nursing studies . 47.1001-1012.

Liu, X.-p., \& Wang, Z.-m. (2013). Perceived risk and organizational commitment: The moderating role of organizational trust. Social Behavior and Personality: An international journal, 41, 229-240.

Rivera, R.R., Fitzpatrick, J.J., Boyle, S.M.(2011). Closing the RN engagement gap: which drivers of engagement matter? J. Nurs. Adm. $41 \quad$ (6), 265- 272, http://dx.doi.org/10.1097/NNA.0b01 3e31821c476c.

Sawatzky, J., \& Enns, C. (2012). Exploring the key predictors of retention in emergency nurses. Journal of Nursing Management. Jul 20(5); 696-707.

Schaufeli, W. B., \& Bakker, A. B. (2004). Test manual for the Utrecht Work Engagement Scale (Unpublished Thesis). Utrecht University, the Netherlands.

Schaufeli, W. B., \& Salanova, M. (2011). Work engagement: On how to better catch a slippery concept. European Journal of Work and Organizational Psychology, 20, 39-46.

Schaufeli, W., Wilmar, B., Bakker, A., \& Arnold, B. (2010). Defining and measuring work engagement. Bringing clarity to the concept. Work engagement: a handbook of essential theory and research. New York: Psychology Press.

Simpson, M.R. (2009). Predictors of work engagement among medicalsurgical registered nurses. Western Journal of Nursing Research. 31(1), 44-65.

Vinje, H.F., \& Mittlemark, M.B., (2008) Community nurses who thrive: the critical role of job engagement in the face of adversity. Journal for Nurses in Staff Development.24 (5), 195202.

Vegsund, H., (2014) work engagement among nurses. Applied Nursing Research, 29, 210-221.

Walker, L. O., \& Avant, K. C. (2011). Strategies for theory construction in nursing (5th ed.). Boston: Prentice Hall.

Wong, C.A., Spence Laschsinger, H.K., \& Cummings, G.G. (2010).Authentic leadership and nurses' voice behaviour and perceptions of care quality. Journal of Nursing Management .18, 889-900. 